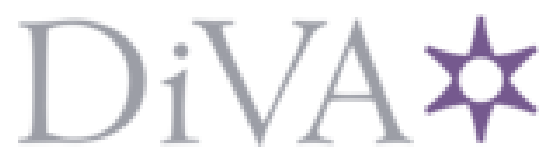

http://www.diva-portal.org

\title{
Preprint
}

This is the submitted version of a paper presented at Micro Electro Mechanical Systems (MEMS), 2017 30th IEEE International Conference on.

Citation for the original published paper:

Errando-Herranz, C., Colangelo, M., Ahmed, S., Björk, J., Gylfason, K B. (2017)

MEMS tunable silicon photonic grating coupler for post-assembly optimization of fiber-to-chip coupling.

In: Institute of Electrical and Electronics Engineers (IEEE) (ed.), Micro Electro Mechanical Systems (MEMS), 2017 30th IEEE International Conference on (pp. 293-296). Institute of Electrical and Electronics Engineers (IEEE)

https://doi.org/10.1109/MEMSYS.2017.7863399

N.B. When citing this work, cite the original published paper.

"(c) 2017 IEEE. Personal use of this material is permitted. Permission from IEEE must be obtained for all other users, including reprinting/ republishing this material for advertising or promotional purposes, creating new collective works for resale or redistribution to servers or lists, or reuse of any copyrighted components of this work in other works."

Permanent link to this version:

http://urn.kb.se/resolve?urn=urn:nbn:se:kth:diva-201140 


\section{MEMS TUNABLE SILICON PHOTONIC GRATING COUPLER FOR POST-ASSEMBLY OPTIMIZATION OF FIBER-TO-CHIP COUPLING}

Carlos Errando-Herranz*, Marco Colangelo, Samy Ahmed, Joel Björk, and Kristinn B. Gylfason

KTH Royal Institute of Technology, Stockholm, SWEDEN

\begin{abstract}
We experimentally demonstrate the first MEMS tunable photonic fiber-to-waveguide grating coupler, and apply it to electrostatically optimize the light coupling between an optical fiber and an on-chip silicon photonic waveguide. Efficient and stable fiber-to-chip coupling is vital for combining the high optical quality of silica fibers with the integration density of silicon photonics. Our device has the potential to lower assembly cost and extend device lifetime, by enabling electrical post-assembly adjustments.
\end{abstract}

\section{INTRODUCTION}

The ability of silicon photonics to tightly pack optical functions onto a silicon chip has recently enabled such innovations as active optical cables and optical interconnects [1]. However, optical glass fibers remain unrivaled for transmission over distances longer than a few millimeters. Thus, almost all silicon photonic devices must couple to optical fibers at some stage $[2,3]$.

The large optical mode mismatch between fibers and silicon waveguides makes coupling challenging (Fig. 1a), and is currently addressed by using grating couplers $[3,4]$. As opposed to edge coupling approaches, grating couplers allow both mode size matching and outof-plane light coupling anywhere on the chip surface (Fig. 1b) [5, 6, 7]. However, errors in fiber-tograting alignment incurred during assembly and through long-term drift of glued parts result in significant and variable optical losses, hampering the spread of this technology (Fig. 1b) [8].

Providing active tuning for grating couplers allows operators to electrically correct for these errors postassembly and in-situ, without the service disruption associated with part replacement. Recently, thermal tuning of silicon photonic gratings has been demonstrated, although it was only used for controlling the transmission spectrum [9]. Furthermore, the high steady state power consumption and thermal crosstalk of thermal tuning excludes its use for long term alignment correction.

In contrast to thermal tuning, a promising approach to tune silicon photonic devices is electrostatic tuning using MEMS. This technology combines the low-power actuation of electrostatic tuning and the ease of fabrication and CMOS compatibility of the silicon-on-insulator platform with the good optical performance of crystalline silicon. Recent demonstrations of this technology include MEMS tunable directional couplers as optical broadband switches [10], a suspended ring resonator tuned horizontally with comb-drive actuators [11], or vertically by a suspended cantilever placed on top [12], and a MEMS tunable ring resonator add-drop filter with an embedded cantilever [13]. a)

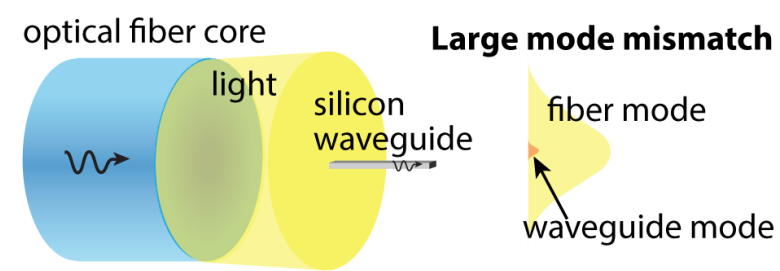

b)

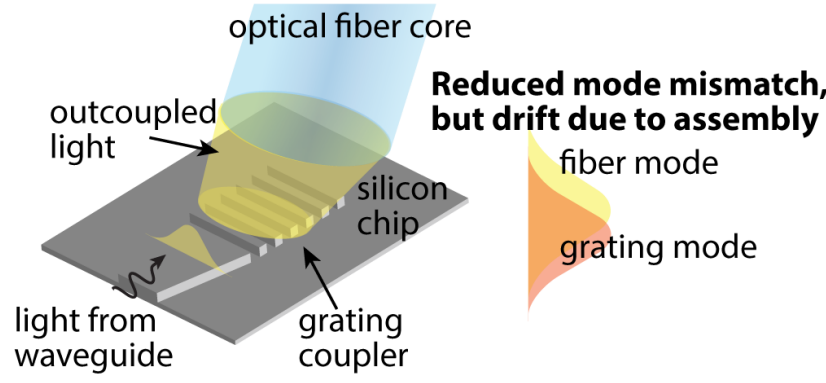

Figure 1: a) Optical fiber-to-chip coupling is key for silicon photonic devices, but the large optical mode mismatch between fibers and silicon waveguides makes coupling challenging. b) This challenge is generally addressed by using waveguide gratings. However, small alignment errors during assembly, and post-assembly drift, affect the coupling efficiency and hamper the spread of this technology.

In this work, we introduce the first MEMS tunable photonic grating coupler, consisting of a silicon on insulator (SOI) cantilever containing a silicon photonic grating tuned by parallel-plate actuation (Fig. 2). This changes the diffraction pattern in the grating, allowing the tuning of the most alignment critical dimension of position along the grating. Our MEMS tunable grating coupler combines the low power consumption of MEMS electrostatic actuation with a very simple fabrication process, to overcome the optical losses resulting from fiber alignment errors and long-term drift of glued fibers.

\section{DESIGN AND FABRICATION}

A schematic of the tunable grating coupler is shown in Fig. 2. The device was fabricated by a very simple SOIbased process using standard photonic SOI wafers (220 nm silicon device layer on $2 \mu \mathrm{m}$ buried $\mathrm{SiO}_{2}$ on a silicon substrate). The fabrication process, presented for the first time in [13], starts by a timed silicon dry etch through an electron-beam patterned mask defining the $110 \mathrm{~nm}$ high silicon ridge waveguides, followed by a second dry etching step through the silicon that defines the cantilever. Then, a wet $\mathrm{SiO}_{2}$ under-etch with hydrofluoric acid (HF) releases the free-standing areas. 


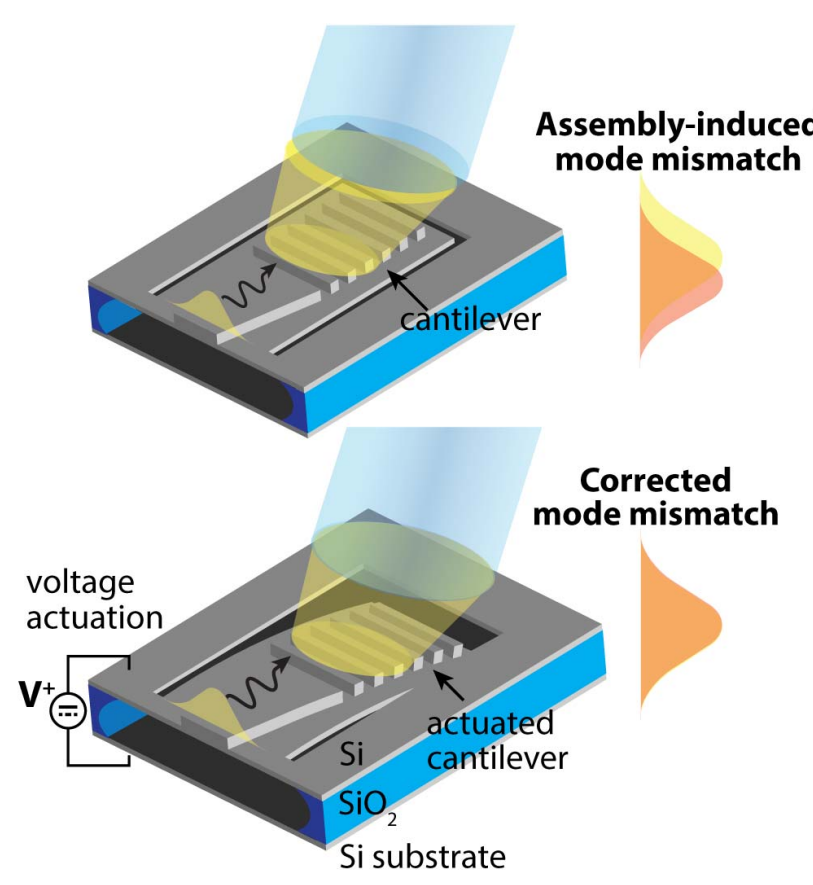

Figure 2. We experimentally demonstrate a MEMS tunable photonic grating, fabricated with a very simple SOI process, for post-assembly optimization of fiber-tochip light coupling. Parallel-plate actuation of the suspended grating affects the diffracted light, allowing tuning of the position of the emitted light beam to match the fiber mode and optimize coupling.

For characterization, the device is optically interfaced via a second static input grating coupler connected to a waveguide that tapers down into a $500 \mathrm{~nm}$ wide singlemode silicon ridge waveguide, and then tapers up again to our tunable grating coupler. The grating couplers were designed using CAMFR $[14,15]$, and optimized for TE light transmission at $1550 \mathrm{~nm}$ wavelength with low backreflection and maximized coupling into a standard singlemode optical fiber tilted 10 degrees with respect to the surface normal, as in [16]. The optimized grating geometry can be found in Table 1 .

\section{RESULTS}

Figure 3 shows an SEM image of our device, showing the silicon ridge waveguide and the under-etched free-standing area containing the tunable grating coupler.

A schematic of the measurement setup is shown in Fig. 4. A diode laser (PGT 201 02, Ericsson) is connected through a fiber polarization controller (FPC03, Thorlabs) to a stripped and cleaved standard telecommunications single mode optical fiber (SMF-28 Ultra, Corning), mounted on a 3 -axis movable stage. The stage holds the fiber with an angle of 10 degrees with respect to the chip surface normal. A second fiber, similarly mounted on a 3axis stage, guides the light from the chip into a photodetector (DET01CFC, Thorlabs), and the resulting photocurrent is measured with an oscilloscope. The actuation voltage is applied between a soft electrical probe in direct contact to the SOI layer and a grounding plate in contact with the silicon substrate.
Table 1: Grating geometry. The grating was optimized for crystalline silicon ridge waveguides with $110 \mathrm{~nm}$ height on a $110 \mathrm{~nm}$ silicon slab, coupling to single-mode silica fibers, with air as top cladding and silica as bottom cladding. The element numbering starts from the end of the waveguide taper.

\begin{tabular}{|c|c|c|}
\hline Element number & Gap [nm] & Tooth [nm] \\
\hline 1 & 448 & 277 \\
\hline 2 & 398 & 297 \\
\hline 3 & 368 & 287 \\
\hline 4 & 348 & 297 \\
\hline 5 & 358 & 297 \\
\hline 6 & 398 & 287 \\
\hline 7 & 378 & 287 \\
\hline 8 & 438 & 307 \\
\hline 9 & 388 & 307 \\
\hline 10 & 348 & 307 \\
\hline 11 & 378 & 297 \\
\hline 12 & 358 & 317 \\
\hline 13 & 368 & 307 \\
\hline 14 & 318 & 327 \\
\hline 15 & 398 & 327 \\
\hline 16 & 338 & 347 \\
\hline 17 & 348 & 307 \\
\hline 18 & 378 & 297 \\
\hline 19 & 398 & 237 \\
\hline 20 & 538 & 327 \\
\hline & & \\
\hline
\end{tabular}

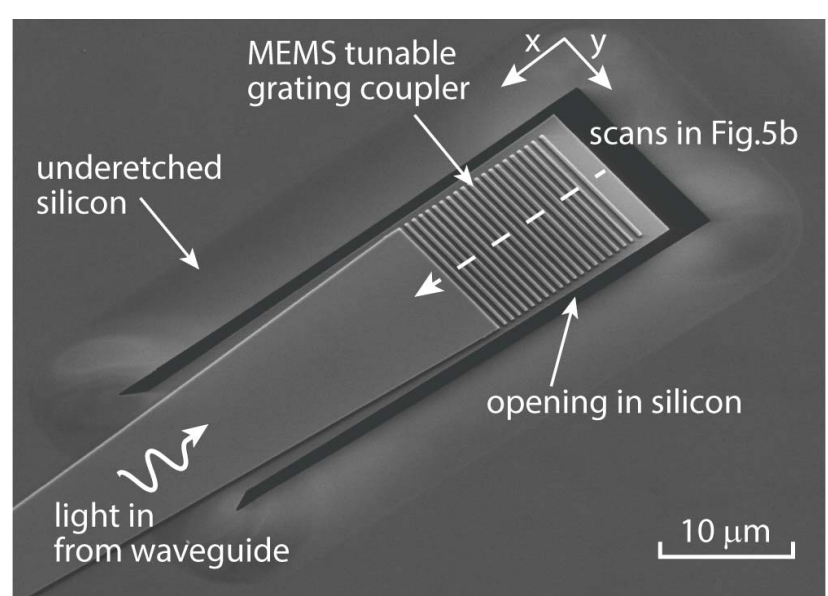

Figure 3. SEM image of our MEMS tunable grating coupler. The axes and the dashed arrow represent the scanning directions in the measurements in Figs. 5 and 6.

The device was characterized using laser light at $1549 \mathrm{~nm}$ and $350 \mu \mathrm{W}$ laser output power coupled into our device via alignment of the input fiber to the static grating coupler. The input light was TE polarized by using a fiber polarization controller. To measure the power distribution coupled out from our tunable grating, the output fiber was rasterized over an area around the grating using motorized stages. We observed a displacement of the optimum coupling position in a two-dimensional scan of the fiber along the $x-y$ plane with increasing actuation voltage (Fig. 5a). 
a)

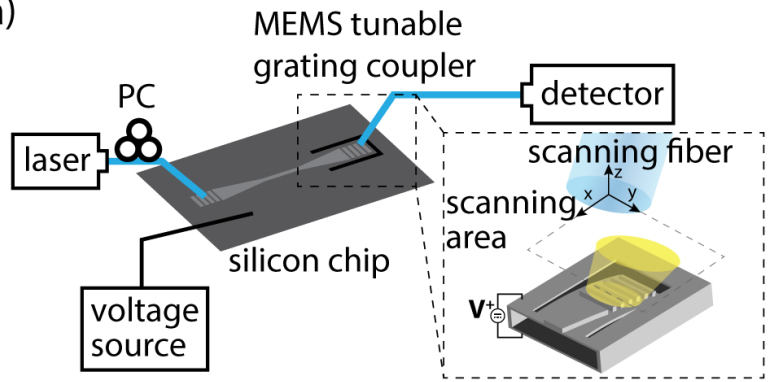

b)

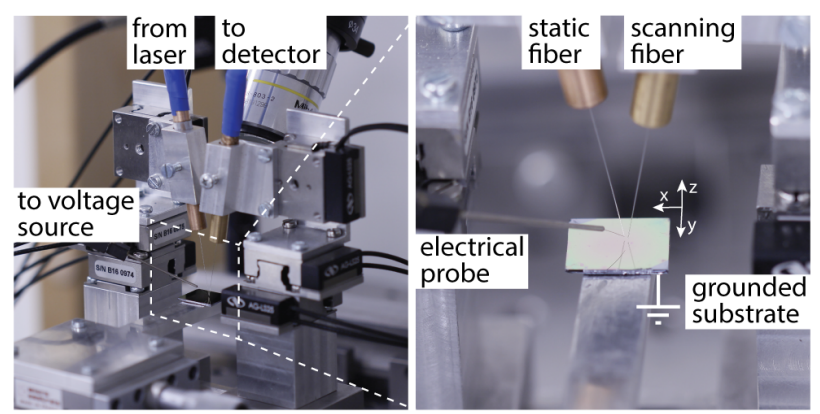

Figure 4. a) Schematic of our measurement setup. Blue lines represent optical fibers. PC stands for polarization controller. Measurements were performed scanning along the MEMS tunable grating with an optical fiber. b) Photographs showing the measurement setup. The fibers are aligned and scanned with 3-axis motorized stages.

Then, the fiber was manually aligned to the optimal position (for $0 \mathrm{~V}$ actuation), and the device was actuated at increasing voltage levels, each of them followed by linear scans along the $\mathrm{x}$ dimension. For each voltage level, three fiber scans were performed to allow for compensation of mechanical drift of the motorized stages. Our results show that voltage actuation up to $6 \mathrm{~V}$ induces a $6 \mu \mathrm{m}$ displacement of the optimum coupling position, combined with an increase in coupling efficiency up to $42 \%$ (Fig. $5 \mathrm{~b}$ ). After returning to a $0 \mathrm{~V}$ actuation voltage, the device remained functional and no pull-in effect was observed. Moreover, we measured a linear tuning rate of $-1.6 \mu \mathrm{m} / \mathrm{V}$ in the actuation voltage range from 2.5 to $6 \mathrm{~V}$ (Fig. 6).

\section{CONCLUSIONS}

We have experimentally demonstrated the first MEMS tunable grating coupler for post-assembly optimization of fiber-to-chip light coupling. Our device combines the low power consumption of MEMS tuning with a tuning range of $6 \mu \mathrm{m}$ along the critical coupling dimension. These results show that our device is able to compensate for the typical drift caused by glued fiber connections or assembly misalignment of up to $3 \mu \mathrm{m}$ [8] by using standard $5 \mathrm{~V}$ CMOS voltage levels. Our results suggest that the presented technology has the potential to overcome current roadblocks to silicon photonics proliferation.

\section{ACKNOWLEDGEMENTS}

The authors thank Mikael Bergqvist for his help building the setup. This work was funded by the Swedish Research council grants 621-2012-5364 and B0460801. a)

b)
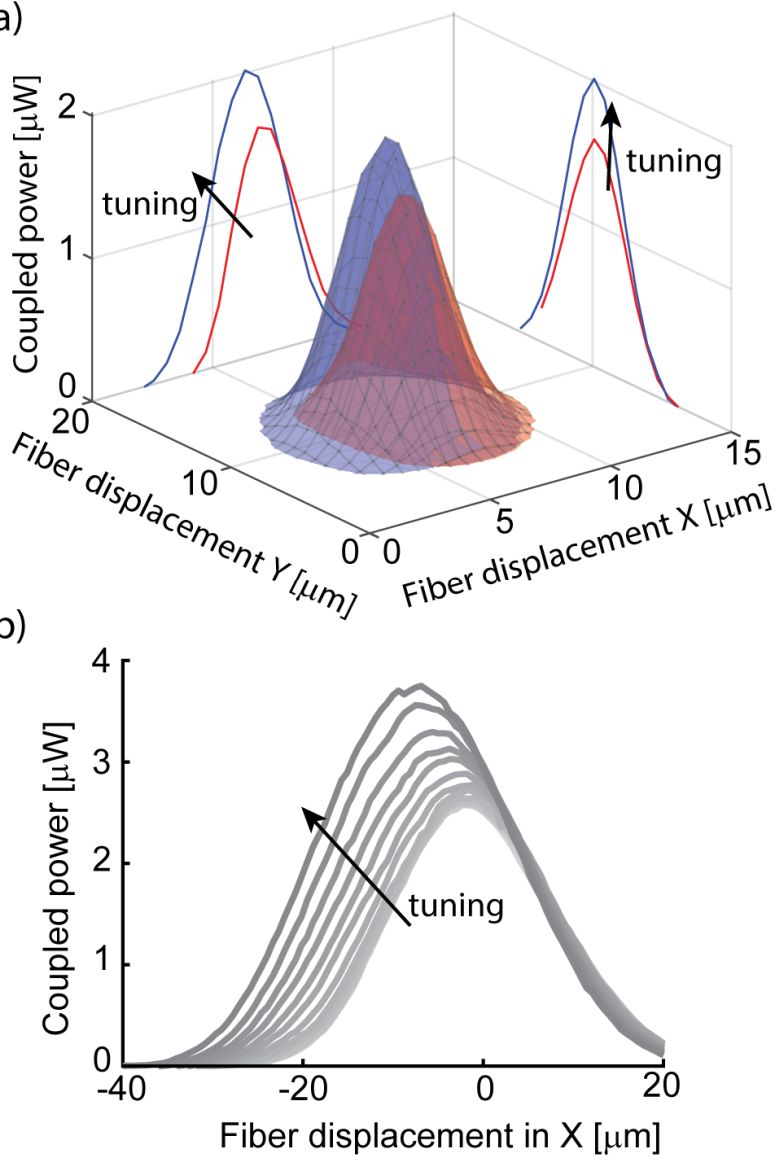

Figure 5. a) Measured optical power coupled into an optical fiber scanning ( $x$ and $y$ directions in Figs. 3 and 4) on top of our device before (red) and after tuning (blue), showing the cross-section planes on both axes at the position with the highest coupled power. b) Linear scans along the $x$ axis for actuation voltages up to $6 \mathrm{~V}$ show a displacement of the optimum coupling position up to 6 $\mu m$.

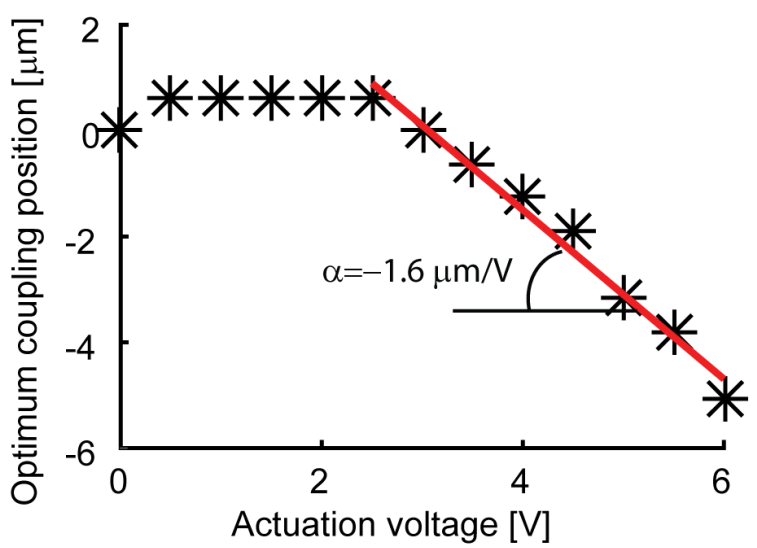

Figure 6. Our MEMS tunable grating coupler achieves a displacement of the optimum coupling position of $6 \mu \mathrm{m}$ with actuation voltage up to $6 \mathrm{~V}$, with a linear tuning range of $-1.6 \mu \mathrm{m} / \mathrm{V}$. 


\section{REFERENCES}

[1] C. Sun, M.T. Wade, Y. Lee, J.S. Orcutt, L. Alloatti, M.S. Georgas, A.S. Waterman, J.M. Shainline, R.R. Avizienis, S Lin, et al. "Single-chip microprocessor that communicates directly using light," Nature, vol. 528, no. 7583, pp. 534-538, Dec. 2015.

[2] D. A. B. Miller, "Device Requirements for Optical Interconnects to Silicon Chips," Proceedings of the IEEE, vol. 97, no. 7, pp. 1166-1185, 2009.

[3] R. Soref, "The Past, Present, and Future of Silicon Photonics," IEEE Journal of Selected Topics in Quantum Electronics, vol. 12, no. 6, pp. 1678-1687, Nov. 2006.

[4] M. Lipson, "Overcoming the limitations of microelectronics using Si nanophotonics: solving the coupling, modulation and switching challenges," Nanotechnology, vol. 15, no. 10, p. S622, 2004.

[5] D. Taillaert et al., "An out-of-plane grating coupler for efficient butt-coupling between compact planar waveguides and single-mode fibers," IEEE Journal of Quantum Electronics, vol. 38, no. 7, pp. 949-955, Jul. 2002.

[6] L. Vivien, D. Pascal, S. Lardenois, D. Marris-Morini, E. Cassan, F. Grillot, S. Laval, J.-M. Fédéli, and L.E. Melhaoui, "Light Injection in SOI Microwaveguides Using High-Efficiency Grating Couplers," J. Lightwave Technol., JLT, vol. 24, no. 10, pp. 3810 3815, Oct. 2006.

[7] A. Mekis, S. Gloeckner, G. Masini, A. Narasimha, T. Pinguet, S. Sahni, and S. Dobbelaere. "A GratingCoupler-Enabled CMOS Photonics Platform," IEEE Journal of Selected Topics in Quantum Electronics, vol. 17, no. 3, pp. 597-608, May 2011.

[8] L. Zimmermann, G. B. Preve, T. Tekin, T. Rosin, and K. Landles, "Packaging and Assembly for Integrated Photonics: A Review of the ePIXpack Photonics Packaging Platform," IEEE Journal of Selected Topics in Quantum Electronics, vol. 17, no. 3, pp. 645-651, 2011.
[9] J. H. Kim et al., "Tunable Grating Couplers for Broadband Operation Using Thermo-Optic Effect in Silicon," IEEE Photonics Technology Letters, vol. 27, no. 21, pp. 2304-2307, 2015.

[10] S. Han, T. J. Seok, N. Quack, B.-W. Yoo, and M. C. $\mathrm{Wu}$, "Large-scale silicon photonic switches with movable directional couplers," Optica, OPTICA, vol. 2, no. 4, pp. 370-375, Apr. 2015.

[11]T. Ikeda and K. Hane, "A microelectromechanically tunable microring resonator composed of freestanding silicon photonic waveguide couplers," Applied Physics Letters, vol. 102, no. 22, p. $221113+$, Jun. 2013.

[12] S. M. C. Abdulla, L.J. Kauppinen, M. Dijkstra, M.J. de Boer, E. Berenschot, H.V. Jansen, R.M. de Ridder, and G.J.M. Krijnen. "Tuning a racetrack ring resonator by an integrated dielectric MEMS cantilever," Opt. Express, vol. 19, no. 17, pp. 15864 15878, 2011.

[13] C. Errando-Herranz, F. Niklaus, G. Stemme, and K. B. Gylfason, "Low-power microelectromechanically tunable silicon photonic ring resonator add-drop filter," Optics Letters, vol. 40, no. 15, p. 3556, Aug. 2015.

[14] P. Bientsman, "Rigorous and efficient modelling of wavelength scale photonic components." Ph.D. thesis (Ghent University 2001).

[15]P. Bienstman and R. Baets, "Optical modelling of photonic crystals and VCSELs using eigenmode expansion and perfectly matched layers," Optical and Quantum Electronics, vol. 33, no. 4, pp. 327-341, Apr. 2001.

[16] M. Antelius, K. B. Gylfason, and H. Sohlström, "An apodized SOI waveguide-to-fiber surface grating coupler for single lithography silicon photonics," Opt. Express vol. 19, pp. 3592-3598, 2011.

\section{CONTACT}

* C. Errando-Herranz, tel: +46760692156; carloseh@kth.se 